\title{
FEMUS-PLATFORM: A NUMERICAL PLATFORM FOR MULTISCALE AND MULTIPHYSICS CODE COUPLING
}

\section{G. BARBI ${ }^{1}$, G. BORNIA ${ }^{2}$, D. CERRONI ${ }^{3}$, A. CERVONE ${ }^{4}$, A. CHIERICI $^{1 *}$, L. CHIRCO ${ }^{5}$, R. DA VIÁ ${ }^{4}$, V. GIOVACCHINI ${ }^{1}$, S. MANSERVISI $^{1}$ AND R. SCARDOVELLI ${ }^{1}$}

${ }^{1}$ DIN, Lab. of Montecuccolino, University of Bologna, Via dei Colli 16, 40136 Bologna, IT

${ }^{2}$ Department of Mathematics and Statistics, Texas Tech University, Lubbock, TX, USA

${ }^{3}$ Politecnico di Milano, P.za Leonardo Da Vinci 32, 20133 Milano, IT

${ }^{4}$ ENEA, Via Martiri di Monte Sole 4, Bologna, 40129, Italy

${ }^{5}$ Sorbonne Université, Institut Jean Le Rond d'Alembert, 4 Place Jussieu, Paris, F

*e-mail: andrea.chierici4@unibo.it

Key words: Multiscale problems, Multiphysics platform, Code coupling

\begin{abstract}
Nowadays, many open-source numerical codes are available to solve physical problems in structural mechanics, fluid flow, heat transfer, and neutron diffusion. However, even if these codes are often highly specialized in the numerical simulation of a particular type of physics, none of them allows simulating complex systems involving all the physical problems mentioned above. In this work we present a numerical framework, based on the SALOME platform, developed to perform multiscale and multiphysics simulations involving all the mentioned physical problems. In particular, the developed numerical platform includes the multigrid finite element in-house code FEMuS for heat transfer, fluid flow, turbulence and fluid-structure modeling; the open-source finite volume CFD software OpenFOAM; the multiscale neutronic code DONJON-DRAGON; and a system-scale code used for thermal-hydraulic simulations. Efficient data exchange among these codes is performed within computer memory by using the MED libraries, provided by the SALOME platform.
\end{abstract}

\section{INTRODUCTION}

In this work, we present the multiscale and multiphysics platform FemusPlatform [1] developed at the Department of Industrial Engineering of the University of Bologna. The FemusPlatform has been developed as an environment where several open, research, and commercial numerical codes can be run together and allows modeling complex physical phenomena on different physical scales. The platform includes the multigrid finite element 
code $\mathrm{FEMuS}$, which is based on a $\mathrm{C}++$ main program that handles several external open-source libraries, such as the Libmesh and PETSc libraries. Libmesh is a $\mathrm{C}++$ finite element library used in the code FEMuS to generate and handle numerical meshes with multiple level refinements [2]. PETSc is a $\mathrm{C}++$ library for linear and non-linear algebra developed using LASPack codes written in Fortran and other solvers [3]. The FEMuS code contains solvers for many different physical problems.

The developed numerical platform is based on the open-source SALOME platform [4], using an approach similar to the NURESAFE platform, developed by the CEA [5]. We use the open-software SALOME platform to add new codes and develop coupling interfaces compatible with open and closed source codes. The SALOME platform brings several tools: KERNEL, GUI, GEOM, SMESH, MED, and PARAVIS module. The KERNEL module provides a common shell for all components, which can be integrated into the SALOME platform. The GUI module provides visual representation with basic widgets and the GEOM module draws and optimizes geometrical models. The SMESH module generates meshes on geometrical models previously created or imported by the GEOM component, PARAVIS performs data visualization and post-processing and finally MED allows to work with highly compressed files.

Several codes can be integrated into the platform, e.g. the open-source finite volume CFD software OpenFQAM

the system-scale code cessfully integrated problems. Some of merical simulation and UNIBO, to simuld
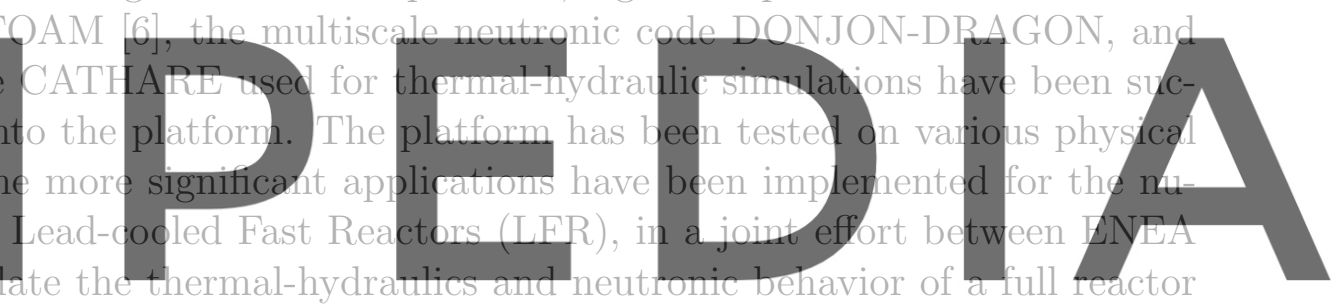

through the coupling between the codes integrated into the numerical platform.

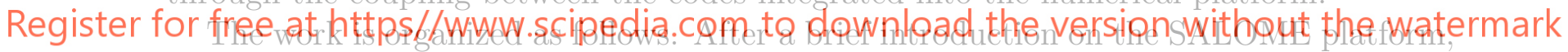

the coupling strategy between all the involved codes is introduced. Then, an example of numerical code coupling between FENIUS and OpenFOAM is discussed, providing guidelines for efficient and optimal data exchange.

\section{FEMuS FEM LIBRARY}

The numerical platform introduced in this work derives from the multigrid finite element library FEMuS. Such a library has been developed at the Department of Industrial Engineering of the University of Bologna, and contains several solvers for incompressible Navier-Stokes equations, heat transfer, turbulence models, Fluid-Structure Interaction problems, multi-phase flows and optimal control with adjoint method.

The FEMuS FEM library has been integrated with the SALOME platform by writing a MEDMem $\mathrm{C}++$ interface to be able to couple the three-dimensional computation obtained with the finite element library with system codes (e.g. CATHARE, RELAP, etc.) or other CFD codes. The interface between the MEDMem and the FEMuS libraries consists basically of three classes: FEMuS, EquationSystemsExtendedM and MeshExtendedM. 
All these classes are located in the src and include directories of the FEMuS library. The FEMuS class is the interface between the library FEMuS and the SALOME platform. The FEMuS interface allows passing commands from the MEDMem library to the EquationSystemsExtendedM class which is the problem solver core. To interact with the FEMuS library two existing FEMuS classes have been extended: the MGEquationsSystem and the MGMesh. The extensions are simply named as EquationsSystemExtendedM and MeshExtendedM, respectively.

The EquationSystemsExtendedM class, which uses only MEDMem functions, inherits the EquationSystem which uses only FEMuS functions. The Equationsystem class contains all the assembly and solver of the FEMuS code. The data from the FEMuS class can be transferred into the assembly routine by a dynamic cast operator, that allows to use child class functions from the parent class. Data can also be transferred in the opposite direction from the EquationSystem to the MEDMem interface by standard $\mathrm{C}++$ inheritance rules.

The MGMesh class contains the multilevel mesh in FEMuS format. To interface FEMuS with MEDMem library a new mesh format should be introduced and the mesh class should be extended. The MeshExtendedM class, which uses only MEDMem functions, inherits the MGMesh which uses only libMesh functions [2]. In the FEMuS library, differ-

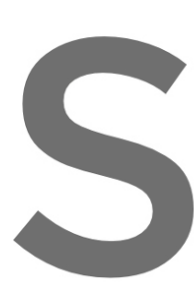
ently from LibMesh library class. Therefore, the interface
MeshExtendedM class. As m
ferred by using a dynamic cast
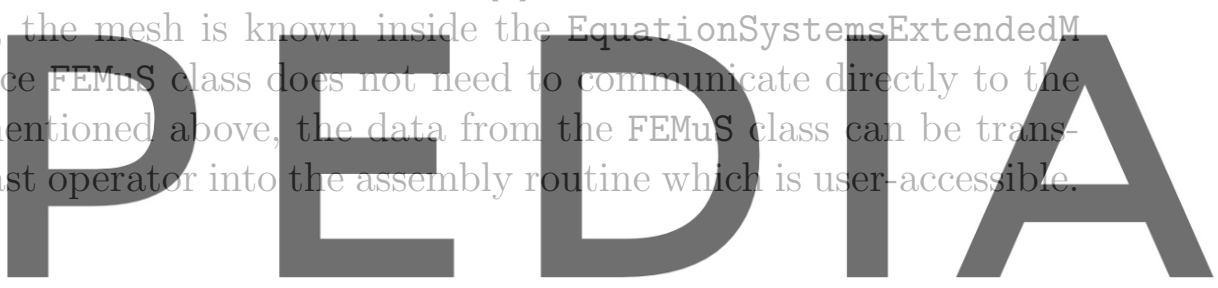

Register for free at https//www.scipedia.com to download the version without the watermark

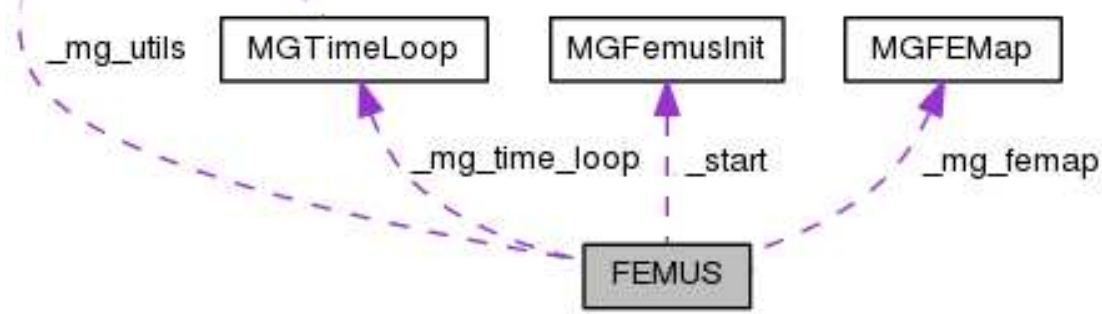

Figure 1: FEMuS class data to communicate with the FEMuS library.

The FEMuS class is the unique interface class. It contains data from both FEMuS and MEDMem libraries to transfer data from one mesh to another (e.g. from FEMuS mesh format, MGMesh, to the MED format). Thus, inside this class there are both pointers mg_mesh and _med_mesh to their respective MGMesh and MED formats. The FEMuS class needs information to extract data from these different mesh structures. In particular, as 
shown in Figure 1, the classes MGUtils, MGFEMap and MGFEMuSinit are available inside FEMuS to transfer basic information such as file names, parameters, multigrid level, fem elements, etc. The MGUtils contains all the file names and input parameter values. The MGFEMap contains information about the finite element mesh structure and MGFEMuSinit class is the manager class for MPI and multigrid solvers.

\section{THE NUMERICAL PLATFORM}

The numerical platform SALOME introduced above has been developed by Commissariat à lénergie atomique (CEA) and Électricité de France (EDF) to provide an advanced open-source platform for Computer-Aided Engineering (CAE) purposes. The platform is composed of several modules, such as KERNEL, GEOM, MESH, PARAVIS, and MED, that can be used for integrating external codes. These modules have been previously described.

We focus now on the MED module, which provides a standard for storing and recovering computer data associated with numerical meshes and fields and facilitates the exchange between codes and solvers. This module is used to read a numerical field from file, and store it in the memory by using the MEDMem library. Moreover, the library provides a complete set of functions, used to access, modify, create meshes and perform operations

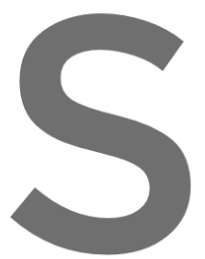
on fields. Note that with the NIED me
codes can be performed at the menory
access on the hard disk.
3.1 SAIONE interface
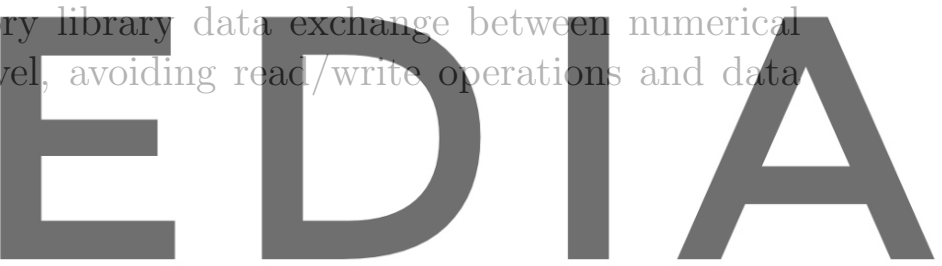

A numerical interface has been developed to couple all the involved codes inside the

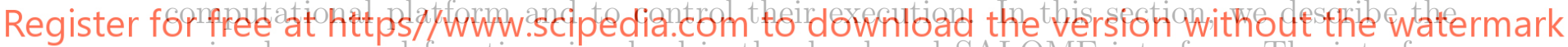
main classes and functions involved in the developed SALOME interface. The interface can be divided into different classes. A driver class, belonging to the top level of the interface, has been implemented to communicate directly with the SALOME platform. The problem class contains three basic functions: setType, setMesh and solve. The setType command sets the physical problem to be solved for each involved code (e.g. Navier-Stokes, energy, turbulence, etc.). The setMesh command sets and prepares the mesh that should be available in both MED and code-specific formats for data exchange. The solve command controls the solution of the discrete system. The equations class inherits the system's particular class which contains the assembly and solver of the generic code. In this class, the solution is accessible, and a field can be extracted or set by using methods class with setField and getField functions. Finally, the grids class is an extension of the mesh class and allows the exchange of data between the code-specific format and the MED library. In Figure 2 we show the numerical scheme implemented to couple the two codes Code 1 and Code 2 in the developed platform. In particular, the two codes are communicating in a process where a numerical field is extracted from 


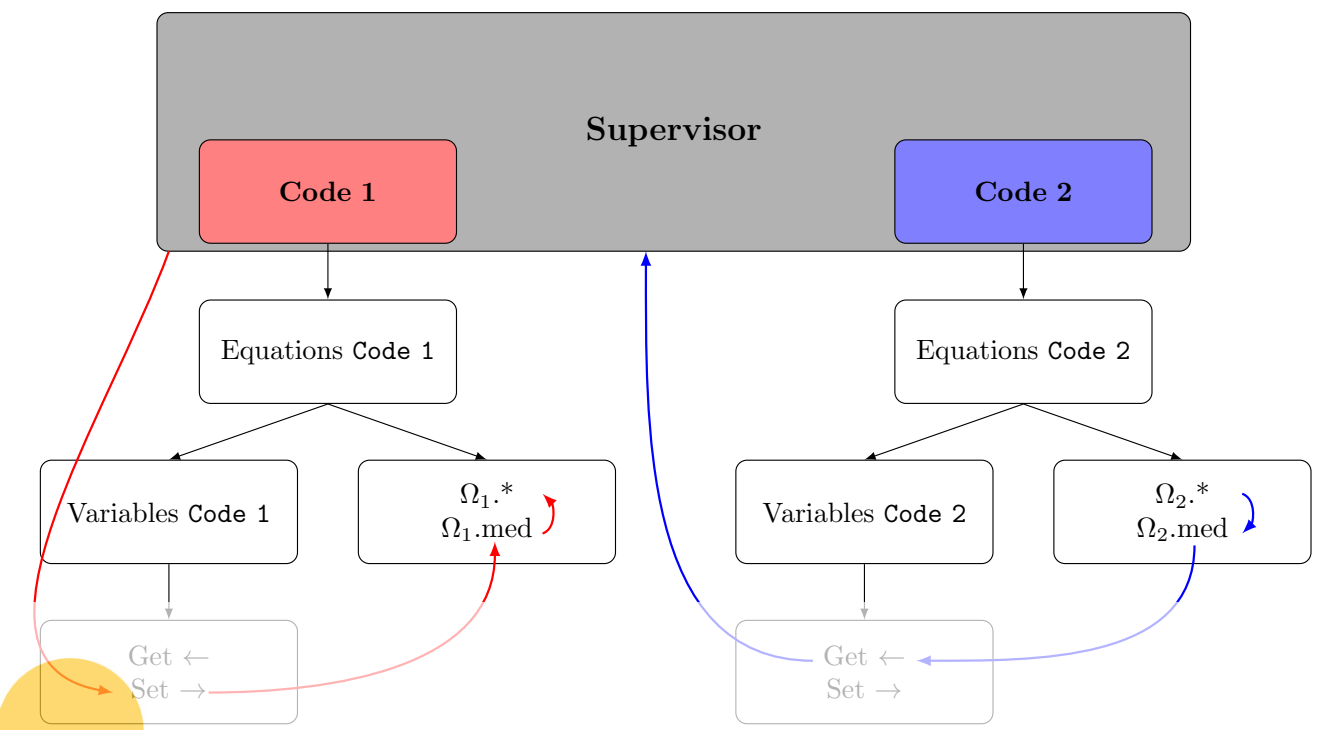

Figure 2: Example of coupling between two different codes (Code 1 and Code 2) controlled by a supervisor.

Code 2 and projected and used into Code 1. The solution is read from the mesh $\Omega_{2}$ * of

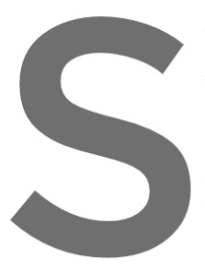
the Code 2 and duplicated in the function Get. Th memory object and can be pas projected on a MED The presented function
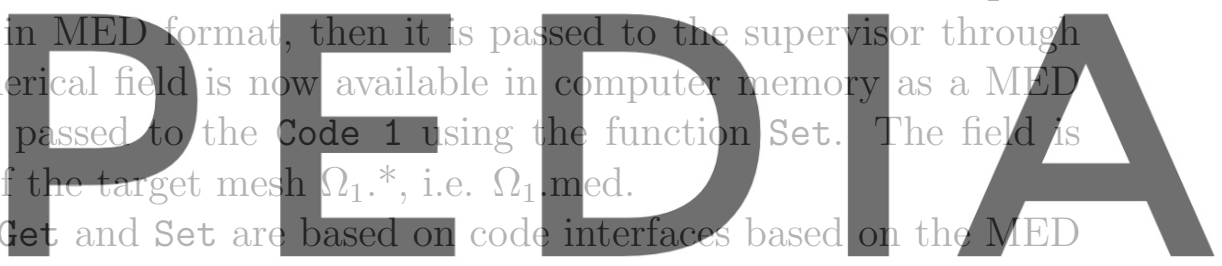

format. In particular, an interface is created on a volume or boundary portion of the

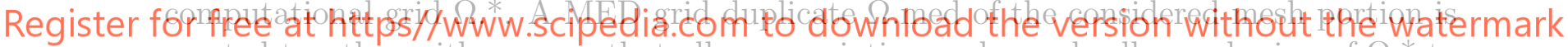
created together with maps $\omega$ that allow associating nodes and cells numbering of $\Omega$.* to nodes and cells numbering of $\Omega$.med. For more information on the developed SALOME interface, the interested reader can consult [7].

\subsection{Data manipulation and projection}

The coupling between different codes can be implemented by using interpolation methods to project the field between different meshes. The used MED library offers several functions for data manipulation and interpolation. In particular, interpolation functions are provided for the piece-wise $P 0$ and node-wise $P 1$ fields. Thus, many algorithms are provided by the MED library to interpolate between $P 0$ and $P 0, P 0$ and $P 1, P 1$ and $P 1$. Moreover, we added a class for the node-wise interpolation $P 2$. Therefore, it is now possible to interpolate between $P 2$ and $P 2$ fields. Moreover, a transformation of $P 2$ fields into $P 0$ fields has been implemented. We underline that the advantage of using MED data structures is that all the developed classes of the interface are not built for a specific code, but can generally be used wherever the MED library is used. 
Node-wise field projection We illustrate now the node-wise field projection technique implemented to project fields between different codes. Let $\Omega_{s}$ and $\Omega_{t}$ be the source and the target mesh divided into $\mathcal{N}_{s}^{e}$ and $\mathcal{N}_{t}^{e}$ finite elements, respectively. Also, let $\psi_{s}$ and $\psi_{t}$ be the source and the target fields. The projection relies on a point search algorithm, in order to find the element $\Omega_{s}^{e}$ in the source mesh containing every node $x_{j}^{t}$ of the target mesh. Then a reconstruction step is needed, aimed at locating the $x_{j}^{t}$ node into the canonical element associated with $\Omega_{s}^{e}$.

The interpolation takes place on the canonical element, so the value of $\psi_{t}$ on node $x_{j}^{t}$ can be determined through the value of $\psi_{s}$ on the nodes of $\Omega_{s}$ and the corresponding interpolation weights. Therefore, if we define $\Psi_{s}$ as the array containing the $\psi_{s}$ values, and $\Psi_{t}$ as the array containing the $\psi_{t}$ values, we have

$$
\Psi_{t}=|P| \Psi_{s},
$$

where $|\boldsymbol{P}|$ is the projection operator. We define now the linear transformation that transforms the local canonical element into the original element as $\pi=\sum_{j=1}^{n_{e}} \boldsymbol{x}_{j}^{e} \varphi_{j}^{e}\left(\boldsymbol{\xi}_{j}^{e}\right)$, where $\boldsymbol{\xi}_{j}^{e}$ are the coordinates of the points in the local reference frame, $\varphi_{j}^{e}(\boldsymbol{\xi})$ are the approximation functions and $n_{e}$ is the number of nodes that composes the $e$-th element. Thus, under the presented hypotheses, the projection operator can be defined as
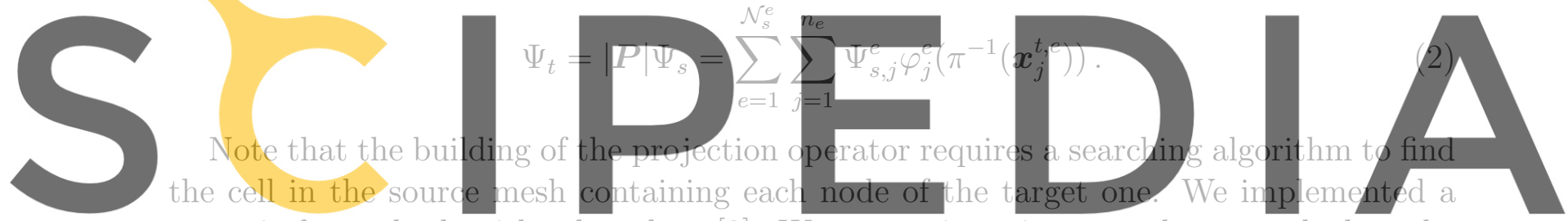

numerical search algorithm based on [8]. We use an iterative procedure to calculate the

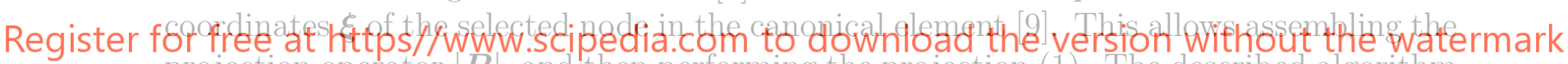
projection operator $|\boldsymbol{P}|$, and then performing the projection (1). The described algorithm can be used to project numerical fields with both $P 1$ or $P 2$ approximations.

Galerkin field projection We also implemented the Galerkin node-wise field projection technique [10]. In this work, we report only a brief sketch of the Galerkin field projection implementation. For more information on the implemented algorithm in the numerical platform one can refer to [7].

The Galerkin technique relies on the fact that the interpolated field $\Psi_{t}$ is the best approximation in $L^{2}$-norm, as

$$
\left\|\Psi_{s}-\Psi_{t}\right\|=\min _{\Psi \in \operatorname{span}\left\{\varphi_{t}\right\}}\left\|\Psi_{s}-\Psi\right\|_{L^{2}} .
$$

With Galerkin projection method the target field is approximated through the weighted interpolation

$$
\Psi_{t}(\boldsymbol{x})=\sum_{j=1}^{\mathcal{N}_{t}} w_{j}(\boldsymbol{x}) \sum_{k=1}^{n_{e}^{s}} \Psi_{s, k}^{e}(\boldsymbol{x}) \varphi_{k}^{e}(\boldsymbol{x})
$$


where $w_{j}(\boldsymbol{x})$ are the used weights. The source mesh element containing the $j$-th target mesh node has been labeled with superscript $e$, and it contains $n_{e}^{s}$ nodes upon which the basis functions $\varphi_{k}^{e}(\boldsymbol{x})$ are defined.

In general, the pointwise technique is useful to project fields from a coarse mesh to a finer one. Conversely, the Galerkin technique is used to project fields from a fine mesh to a coarser one.

\section{RESULTS}

In this section, some simple numerical results are reported to show the effectiveness of the implemented coupling platform. In particular, the integration technique used to couple CFD and system codes is tested, as well as the projection algorithm to couple two CFD codes. Finally, a simple coupling between the FEMuS code and OpenFOAM is presented.

\subsection{Coupling between system and CFD codes}

The coupling between a system code and the FEMuS finite element library has been presented in past works, and the interested reader can consult $[11,12]$. In particular, the FEMuS library has been coupled with the system code CATHARE to have multiscale

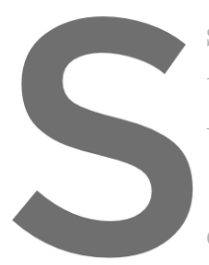
simulations of complex the program is runni writing fields on exter

The coupling betwe of them, the CFD code
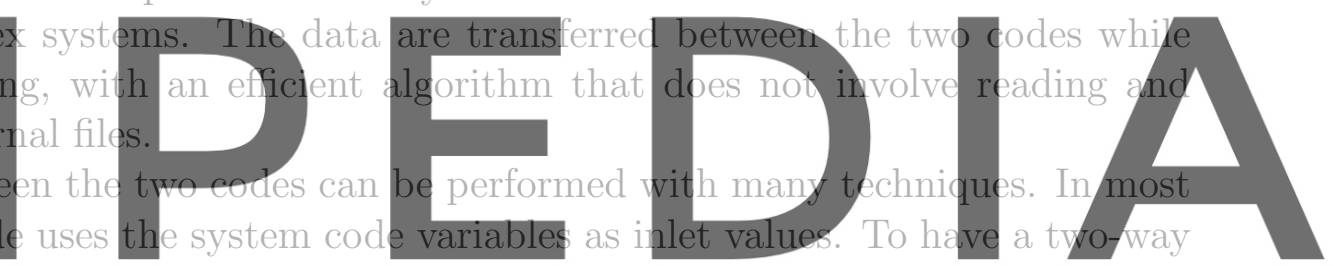

coupling, the average value of the solution at the outlet of the CFD code's domain is used

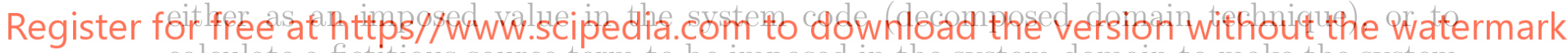
calculate a fictitious source term to be imposed in the system domain to make the system code solution equal to the CFD one (overlapping domain technique, see [11]). Thus, we implemented a function able to build a MED interface on a desired portion of the domain, containing all the solution fields on it. Then, a function able to integrate all the variables on a given MED interface based on a classical Gauss integration has been developed as

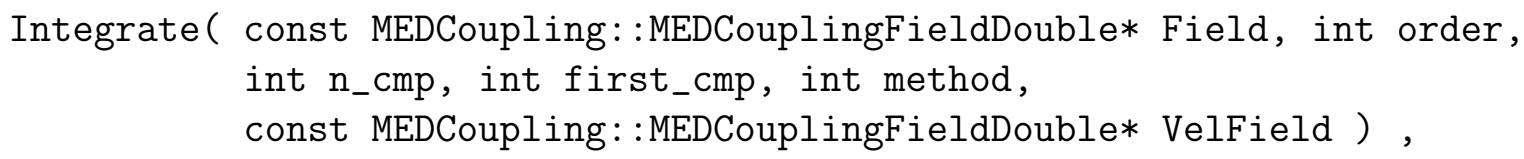

where Field is the MED solution field on the specified interface and method can be method $=$ mean for a classical integration divided by the value of the area, method $=$ aximean for axisimmetric integrations, method $=$ bulk for integrals weighed to the velocity field (with velocity VelField), etc. The user can integrate $\mathrm{n}_{-} \mathrm{cmp}$ solution components, starting from the component first_cmp, with interpolation order given by order.

To test this function, we consider a quadrangular domain $\Omega=\{x \in[0,1], y \in[0,1]\}$, and initialize an interface on the lower boundary of the domain $\left(I_{\Gamma}\right)$ and an interface on 
the whole domain $I_{\Omega}$. We impose some simple polynomial fields $\mathcal{F}(x, y)$ on $\Omega$ and compare the results of the Integrate function calculated both on $I_{\Gamma}$ and $I_{\Omega}$ with the analytical results.

Table 1: Comparison between the numerical results obtained with the function Integrate and the analytical results.

\begin{tabular}{ccc|ccc}
\hline \multicolumn{3}{c|}{$I_{\Gamma}$} & \multicolumn{3}{|c}{$I_{\Omega}$} \\
\hline $\mathcal{F}(x, y)$ & $\int_{I_{\Gamma}} \mathcal{F}(x, y) d \gamma$ & $A_{\text {res }}$ & $\mathcal{F}(x, y)$ & $\int_{I_{\Omega}} \mathcal{F}(x, y) d \gamma$ & $A_{\text {res }}$ \\
\hline$x$ & 0.5 & 0.5 & $0.5(x+y)$ & 0.5 & 0.5 \\
$x^{2}$ & 0.33333 & 0.33333 & $0.5\left(x^{2}+y^{2}\right)$ & 0.33333 & 0.33333 \\
$x^{3}$ & 0.25 & 0.25 & $0.5\left(x^{3}+y^{3}\right)$ & 0.25 & 0.25 \\
$x^{4}$ & 0.20052 & 0.20000 & $0.5\left(x^{4}+y^{4}\right)$ & 0.20052 & 0.20000 \\
$x^{5}$ & 0.16797 & 0.16667 & $0.5\left(x^{5}+y^{5}\right)$ & 0.16796 & 0.16667
\end{tabular}

In Table 4.1 we report the numerical results of the integral carried out on both $I_{\Gamma}$ and $I_{\Omega}$, for various functions $\mathcal{F}(x, y)$. We also compare the numerical result with the

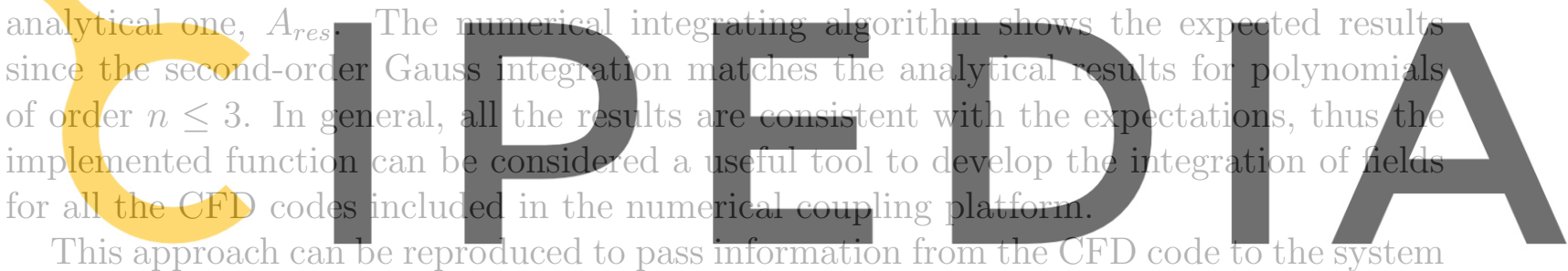

This approach can De reprocuced to pass information trom cone coupling. At the same time, the values can be passed Register for free at https/dwww. scipedia. comp to download the version without the watermark fixed value on a certain MED interface.

\subsection{Coupling between different CFD codes}

The developed numerical platform provides the coupling between different CFD codes, as stated above. Since the information between the codes passes through the MED format, we can couple different numerical schemes (e.g. finite element, finite volume, etc.) or different element orders (e.g. linear and quadratic finite elements). To do so, we implemented various functions to extract a numerical field from a mesh, and project it into a copy of the source mesh in the MED format. Then, as reported in Section 3, we project the field into a copy of the target mesh in the MED format. Lastly, we project the field from the MED format to the target format. In particular, the projection of the field srcField between the two MED meshes is performed through the function

setFieldSource(int interface_name, int $\mathrm{n}_{-} \mathrm{cmp}$, const MEDCoupling: :MEDCouplingFieldDouble* srcField) , 
where $\mathrm{n}_{-} \mathrm{cmp}$ components are projected from the source MED mesh to the MED interface interface_name. Then, the values are projected from the MED format to the target mesh format with the function

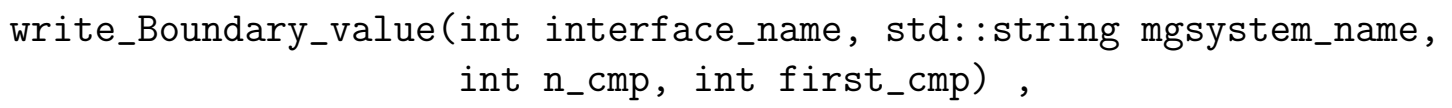

where mgsystem_name is a string which identifies the physical system to be solved.

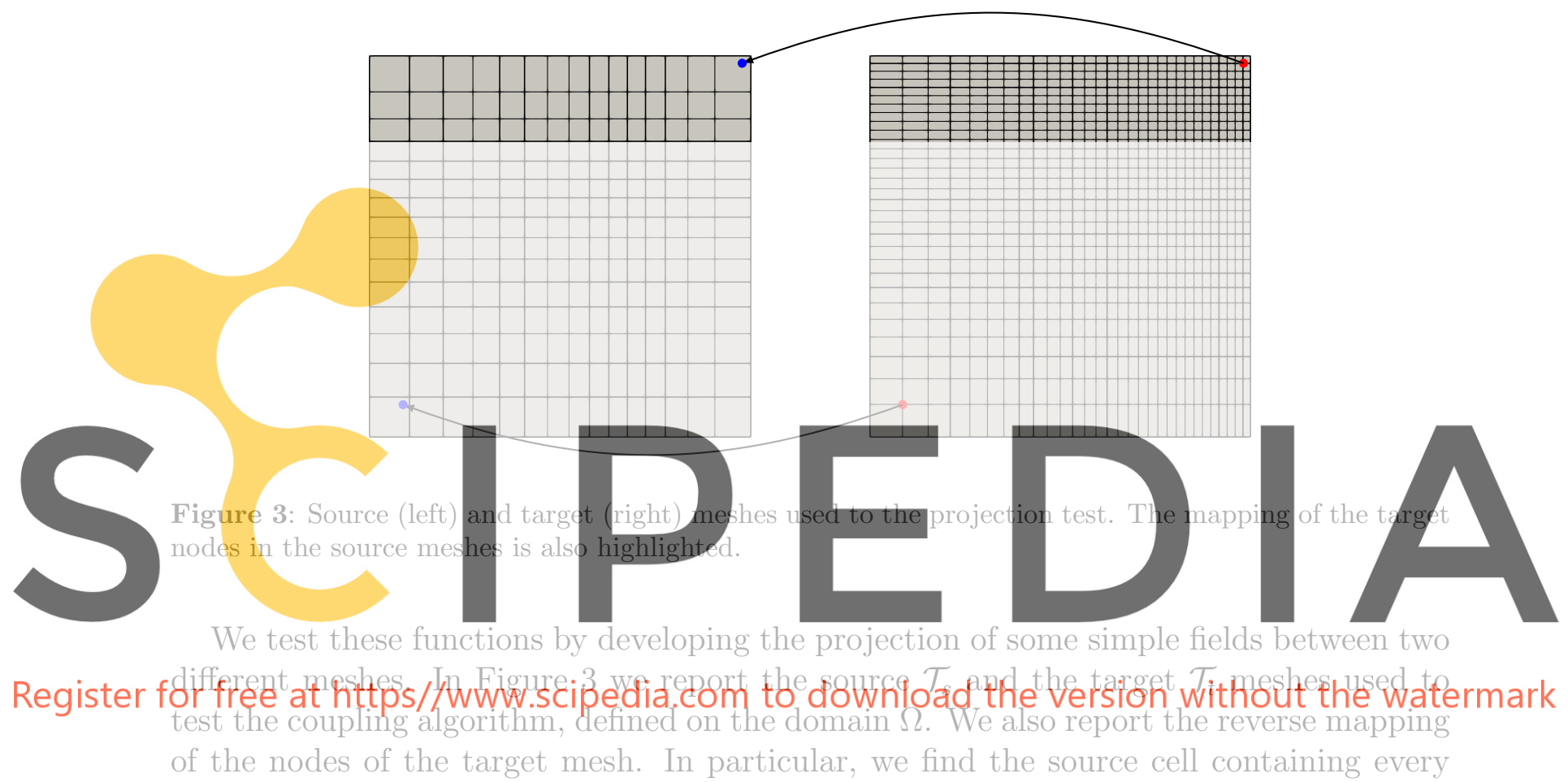

target node (in red in Figure). This search algorithm is the basis of the data projection described in section 3.2 .

We impose different fields $\mathcal{F}(x, y)$ on $\mathcal{T}_{s}$, and we project them on $\mathcal{T}_{s}$. In particular, we consider a quadratic case $\mathcal{F}_{1}(x, y)=(2 x-1)^{2}+(2 y-1)^{2}+0.5$, a quartic case $\mathcal{F}_{2}(x, y)=(2 x-1)^{4}+(2 y-1)^{4}+0.5$, and a sinusoidal function $\mathcal{F}_{3}(x, y)=2-2 \cos (\pi x)$.

In Figure 4 we report the projection between the two presented meshes in the sinusoidal case. Note that the projection algorithm projects the field correctly. To analyze the correct field projection, we report in Table 4.2 the $L^{2}$-norm of the solution calculated over $\Omega$ in all the tested cases. We also report the percentage error between the source and the target fields. Note that the $L^{2}$-norm of the target fields matches the $L^{2}$-norm of the tested source fields, and all the errors are smaller than $1 \%$.

Therefore, the function implemented for the CFD code coupling works properly for the field projection in all the simple cases tested. The implemented functions are designed to project fields between different finite-element and/or finite-volume codes. 

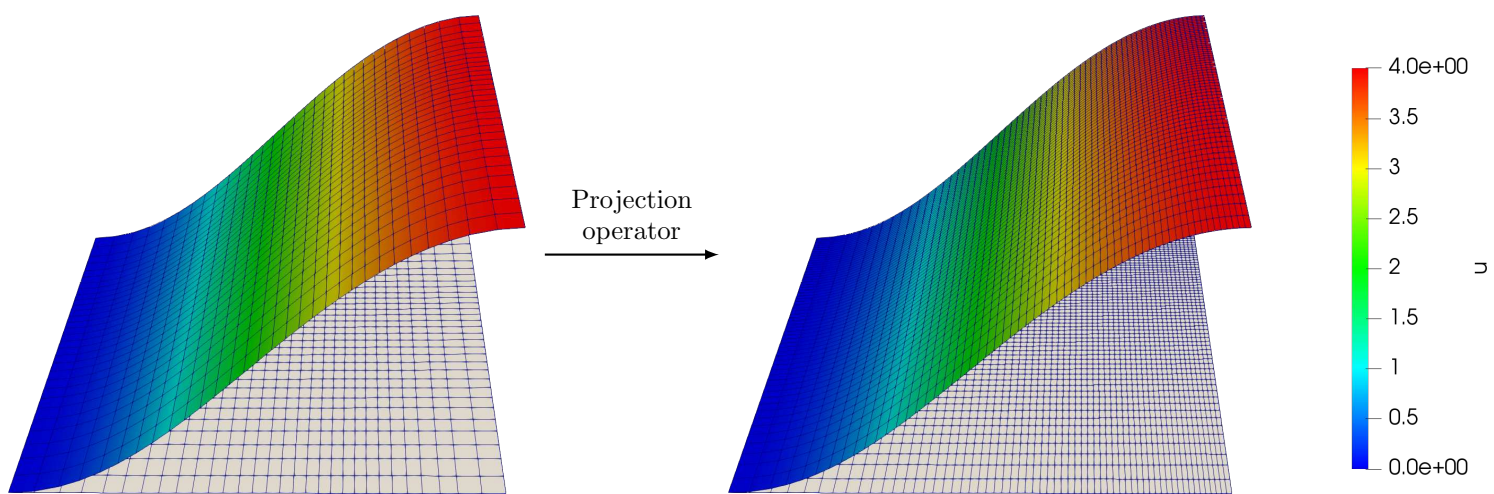

Figure 4: Sinusoidal projected field $\mathcal{F}_{3}(x, y)$ between $\mathcal{T}_{s}$ and $\mathcal{T}_{t}$.

Table 2: $L^{2}$-norm of the source and target solution in all the tested cases. The percentage error is also reported.

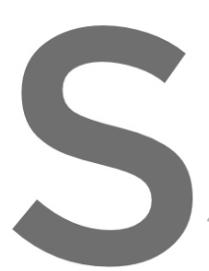

\begin{tabular}{cccc}
$\mathcal{F}(x, y)$ & $\|u\|_{s}$ & $\|u\|_{t}$ & $\varepsilon \%$ \\
\hline $\mathcal{F}_{1}(x, y)$ & 0.9856 & 0.9795 & $0.62 \%$
\end{tabular}

\begin{tabular}{cccc}
$\mathcal{F}(x, y)$ & $\|u\|_{s}$ & $\|u\|_{t}$ & $\varepsilon \%$ \\
\hline $\mathcal{F}_{1}(x, y)$ & 0.9856 & 0.9795 & $0.62 \%$
\end{tabular}

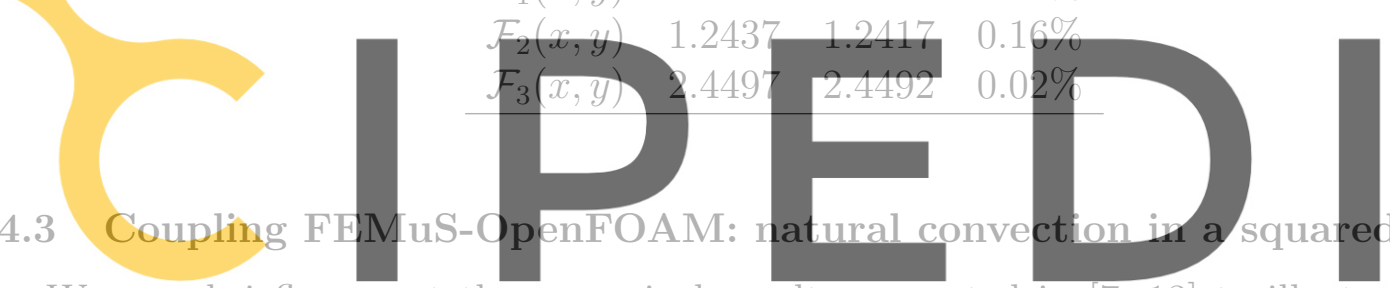

We now briefly report the numerical results presented in $[7,13]$ to illustrate the nu-

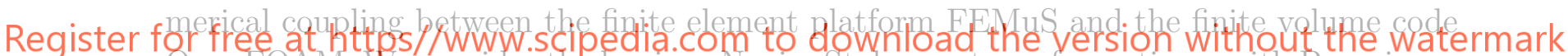
OpenFOAM. We consider the laminar Navier Stokes system of equations, with Boussinesq approximation for the buoyancy term, composed by the continuity equation $\nabla \cdot u=0$, the momentum equation, given by

$$
\rho\left(\frac{\partial \boldsymbol{u}}{\partial t}+\boldsymbol{u} \cdot \nabla \boldsymbol{u}\right)=-\nabla P+\nabla \cdot\left[\mu\left(\nabla \boldsymbol{u}+\nabla \boldsymbol{u}^{T}\right)\right]+\underbrace{\rho \boldsymbol{g} \beta\left(\tilde{T}-T_{\text {ref }}\right)}_{\text {coupling term }},
$$

and the temperature equation $\rho c_{p}\left(\frac{\partial T}{\partial t}+\boldsymbol{u} \cdot \nabla T\right)=k \Delta T$. We consider the domain $\Omega=$ $\{x \in[0, L], y \in[0, L]\}$, and we impose no-slip velocity boundary conditions on the bottom, right and left walls, and tangent velocity condition on the top wall. For the temperature field, we impose insulation boundary conditions on the top and the bottom walls, $T=T_{c}$ Dirichlet boundary condition on the left wall, and $T=T_{h}$ Dirichlet boundary condition on the right wall.

The temperature field is calculated with OpenFOAM, then it is projected to FEMuS for the buoyancy force term calculation. The results are presented in terms of nondimensional variables, namely $x^{+}=x / L, y^{+}=y / L, u^{+}=\frac{\rho c_{p}}{k} u L, v^{+}=\frac{\rho c_{p}}{k} v L$, and 

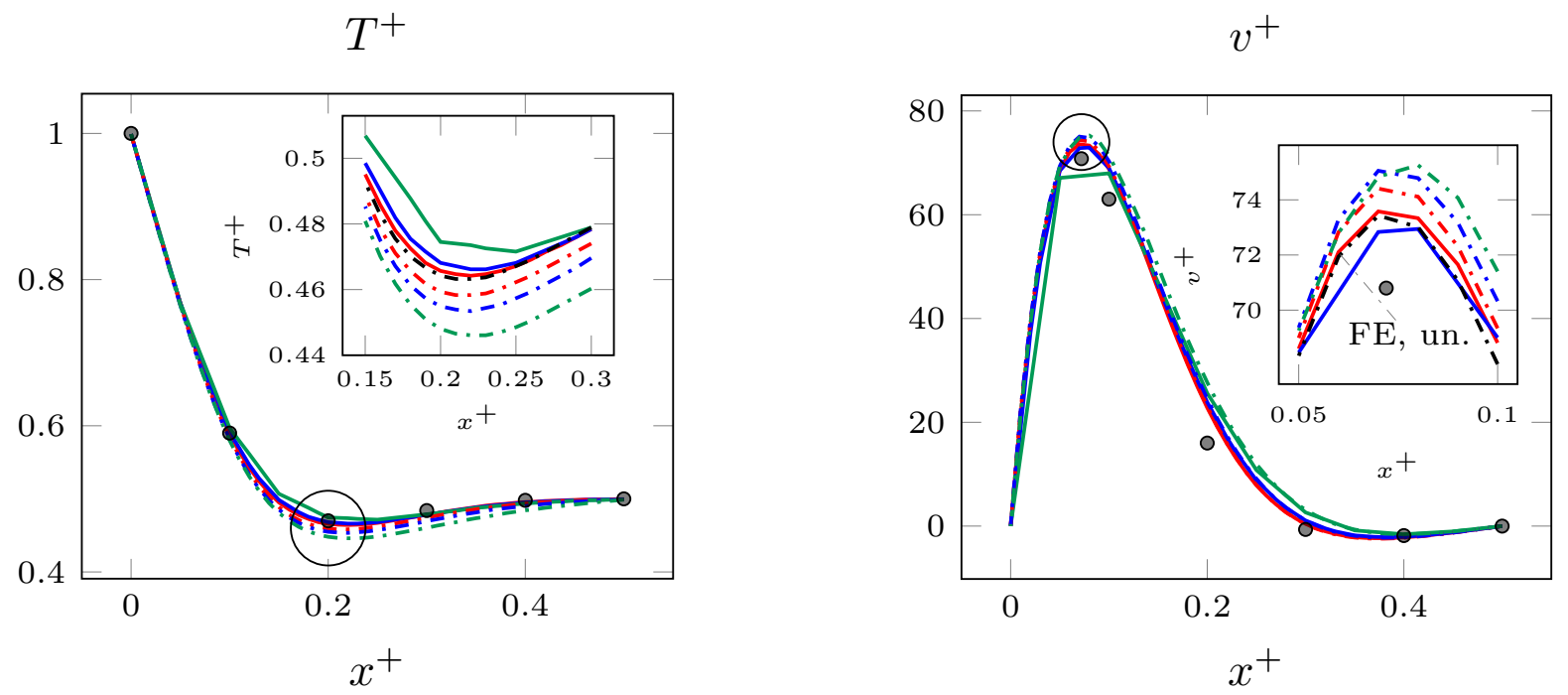

Figure 5: Non-dimensional temperature $T^{+}$(left) and velocity $v^{+}$(right) profiles along $y^{*}=0.5$.

$T^{+}=\left(T-T_{c}\right) /\left(T_{h}-T_{c}\right)$.

In Figure 5 we report the non-dimensional temperature $T^{+}$and velocity $v^{+}$profiles along the line $y^{*}=0.5$. Solid lines stand for OpenFOAM solutions, while dash-dotted lines stand for the solution of the coupling between FEMuS and OpenFOAM. The different used meshes can be identified by the line color: green for the $20 \times 20$ coarse mesh, blue for the $40 \times 40$ mesh, and red for the $80 \times 80$ fine mesh. Dots represent reference values taken from [14].

The presented results show that the coupling between the two codes works properly, and the temperature field is successfully passed from OpenFOAM to FEMuS. The obtained numerical fields match the standalone OpenFOAM results and the reference results.

\section{CONCLUSIONS}

In this work, the numerical platform FemusPlatform has been presented. The platform allows the coupling of several different codes, ranging from one-dimensional system codes to finite element and finite volume CFD codes, in a unified framework. The SALOME platform has been used as a central tool to develop all the routines and classes for the projection and integration of fields through the MED format. The platform can be used for complex multiscale and multiphysics numerical simulations, combining different codes.

The presented preliminary results show that the implemented routines for the integration and projection of numerical fields work properly. Finally, a simple example of numerical coupling between the finite element code FEMuS and the finite volume code OpenFOAM has been reported, showing the proper functioning of the coupling process. 


\section{REFERENCES}

[1] FemusPlatform https://github.com/FEMuSPlatform

[2] Kirk B. S., et al. libMesh: a $C++$ library for parallel adaptive mesh refinement/coarsening simulations. Engineering with Computers 22.3-4 (2006): 237-254.

[3] Balay, Satish, et al. PETSc users manual. (2019).

[4] Bergeaud V. and Lefebvre V. SALOME. A software integration platform for multiphysics, pre-processing and visualisation. (2010).

[5] Chanaron B. Overview of the NURESAFE European Project. Nuclear Engineering and Design 321 (2017): 1-7.

[6] OpenFOAM project. URL:https://www.openfoam.com/

[7] Da Vià R. Development of a computational platform for the simulation of low Prandtl number turbulent flows. (2019).

[8] Löhner R. Robust, vectorized search algorithms for interpolation on unstructured grids. Journal of computational Physics 118.2 (1995): 380-387.

[9] Silva, G.H.C., et al. Exact and efficient interpolation using finite elements shape functions. European Journal of Computational Mechanics 18.3-4 (2009): 307-331.

[10] Farrell P. E. and Maddison J. R. Conservative interpolation between volume meshes by local Galerkin projection. Computer Methods in Applied Mechanics and Engineering 200.1-4 (2011): 89-100.

[11] Chierici A., et al. A multiscale numerical algorithm for heat transfer simulation between multidimensional CFD and monodimensional system codes. Journal of Physics: Conference Series. Vol. 923. No. 1. IOP Publishing, 2017.

[12] Cervone A., et al. Validation of a multiscale coupling algorithm by experimental tests in tall-3D facility. 6th ECCM and 7th ECFD ECCOMAS 2018 conference, 2020.

[13] Da Vià R, et al. Natural convection in a squared cavity via a numerical coupling between a FEM code and OpenFOAM. AIP Conference Proceedings. Vol. 1978. No. 1. AIP Publishing LLC, 2018.

[14] Wan C., et al. A new benchmark quality solution for the buoyancy-driven cavity by discrete singular convolution. Numerical Heat Transfer: Part B: Fundamentals 40.3 (2001): 199-228. 